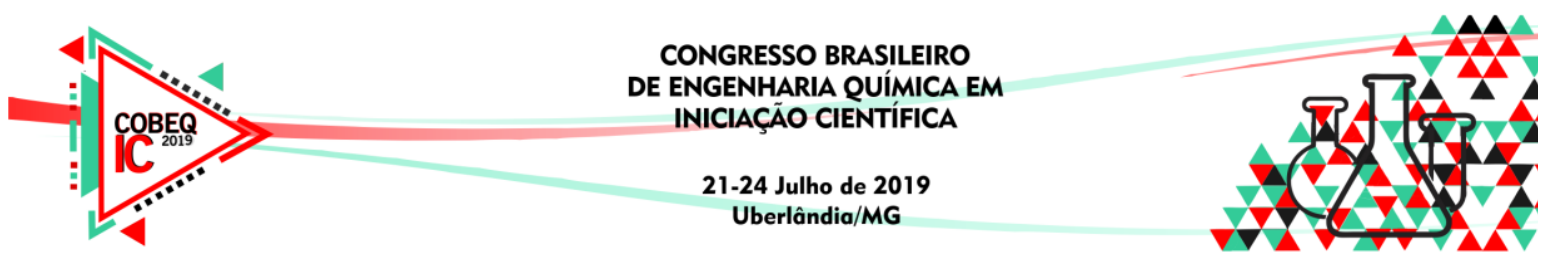

\title{
PREPARAÇÃO DE BIOADSORVENTE E AVALIAÇÃO FLUIDODINÂMICA DA ADSORÇÃO DE CORANTE TÊXTIL EM COLUNA DE LEITO FIXO
}

\author{
DANIEL K. T. ${ }^{1}$, RIBEIRO T. C. ${ }^{1}$, SANTOS E.P.C.C. ${ }^{2}$, BELLIDO J.D.A. ${ }^{1}$ e MELGAR L. Z. ${ }^{1}$ \\ ${ }^{1}$ Universidade Federal de São João del-Rei, Departamento de Engenharia Química \\ ${ }^{2}$ Universidade de São João Del-Rei, Departamento de Tecnologia em Engenharia de Civil, \\ computação e humanidades \\ E-mail para contato: kelenteixeira94@gmail.com
}

\begin{abstract}
RESUMO - A síntese do bioadsorvente foi realizada a partir da casca de arroz, que passou por tratamentos químicos com $\mathrm{NaOH}$ e por tratamento térmico na ausência de oxigênio, formando assim o biochar (biocarvão). O objetivo deste trabalho foi a utilização do biochar em coluna de leito fixo para a remoção do Verde Malaquita. Os ensaios em coluna foram conduzidos avaliando três parâmetros, a concentração da solução de corante, vazão de entrada e altura do leito e para cada experimento foram determinados os tempos de ruptura e saturação, as capacidades de ruptura e saturação, a zona de transferência de massa e o tempo de residência. Com variação da altura do leito, o experimento que combinou uma melhor performance referente a zona de transferência de massa (ZTM) com $0,87 \mathrm{~cm}$ e ao tempo de residência (EBCT) de 294,52 min, foi nas condições de $4 \mathrm{~mL} \cdot \mathrm{min}^{-1}$, altura do leito de 1,5cm e concentração inicial de $20 \mathrm{mg} . \mathrm{L}^{-1}$. Para a variação de concentração observou-se um aumento na ZTM de $0,25 \mathrm{~cm}$ em $15 \mathrm{mg} . \mathrm{L}^{-1}$ para $0,51 \mathrm{~cm}$ em $20 \mathrm{mg} . \mathrm{L}^{-1}$ e uma diminuição dos tempos de ruptura e saturação com o aumento da concentração. A eficiência de remoção do corante foi significativa, com uma média de $80 \%$, o que indica que o biocarvão proveniente da casca de arroz é um possível bioadsorvente alternativo no tratamento de efluentes que contenham esse corante.
\end{abstract}

\section{INTRODUÇÃO}

As indústrias têxteis estão entre as maiores usuárias de água, e por este motivo geram grandes volumes de efluentes. Esses efluentes apresentam forte coloração, devido ao fato de parte do corante não se fixar completamente à fibra. (Dellamatrice et al., 2016).

Dentre os compostos existentes nos efluentes têxteis, os corantes estão entre os mais agressivos ao meio ambiente. A poluição de corpos d'água com estes compostos provocam, além da poluição visual, alterações em ciclos biológicos afetando principalmente processos de fotossíntese (Souza, 2013). Devido às implicações ambientais citadas, tem-se estudado métodos para corrigir os danos gerados e evitar danos futuros. Métodos como tratamento biológico, coagulação, flotação, eletrocoagulação e oxidação já foram explorados para efluentes têxteis, no entanto, em alguns casos são ineficientes, dispendiosos e inviáveis economicamente. Nesse sentido um dos métodos promissores é o tratamento por adsorção, onde se utiliza resíduos agroindustriais como adsorventes (Santhanam et al., 2017). 


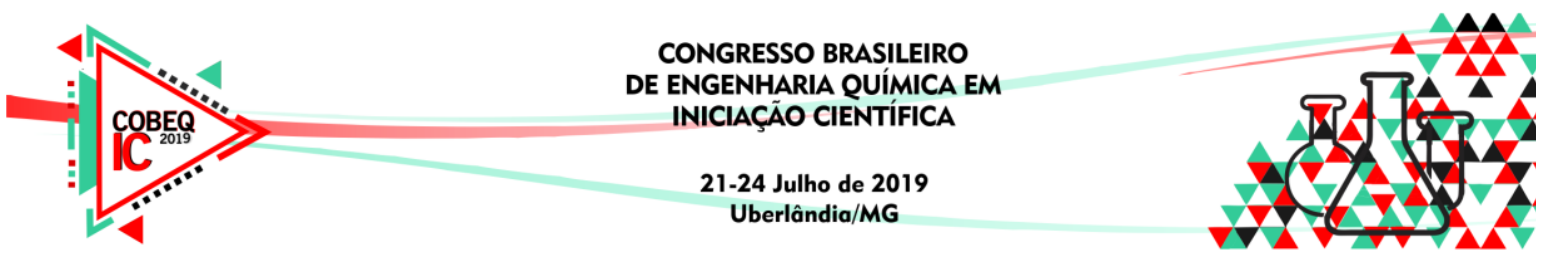

No processo de adsorção em coluna de leito fixo, o adsorvato a ser tratado passa através de um leito empacotado a uma taxa de fluxo constante. A eficiência de um estudo em coluna de leito fixo é baseada na construção da curva de ruptura (breakthrough). Vários fatores, como vazão de alimentação, concentração inicial de soluto, altura do leito e natureza do equilíbrio do processo de adsorção contribuem para a forma que a curva de ruptura apresentará. A zona de transferência de massa (ZTM) é a região dentro da coluna onde a concentração de adsorvato varia entre 5\% e 95\% da concentração inicial, ou seja, é a região onde efetivamente ocorre a transferência de massa do processo de adsorção (Ribas, 2016).

A casca de arroz (CA) é um resíduo oriundo do beneficiamento do arroz. Por ser extremamente abundante o intuito é reutilizar esse material como um bioadsorvente em potencial, uma vez que a sua composição é $32,24 \%$ de celulose, $21,34 \%$ de hemicelulose, $21,44 \%$ de lignina em peso e o restante $(15,05 \%)$ é cinza mineral, água e outros constituintes. (Alexander et al.,2017). A utilização da CA como material adsorvente apresenta vantagens como o baixo custo, facilidade de obtenção, abundância, insolubilidade em água e estabilidade química. (Montanher et al., 2005). O uso de solução de $\mathrm{NaOH}$ na lavagem da casca de arroz tem sido empregado para remoção de substâncias solúveis em meio alcalino, as quais estão presentes na superfície da fibra e interferem no processo de adsorção (Mimura et al., 2010). Também é possível submeter a CA a tratamentos térmicos, nesse caso, uma queima ideal é necessária para o seu uso como adsorvente, ou seja, o material deve apresentar uma estrutura porosa, com alta área específica e diâmetro de poros (Manique, 2011).

O objetivo deste trabalho foi a preparação do material, sua caracterização e a avaliação fluidodinâmica da adsorção do corante Verde Malaquita utilizando o biocarvão da casca de arroz como bioadsorvente em coluna de leito fixo, variando os seguintes parâmetros, altura do leito, vazão e concentração da solução de corante.

\section{MÉTODOS}

Sintetização do Biochar: A sintetização do biochar iniciou-se a partir da lavagem da casca de arroz com água destilada em temperatura ambiente e a $100^{\circ} \mathrm{C}$ e em seguida secagem em estufa a $60^{\circ} \mathrm{C}$ por 24 horas. No tratamento químico utilizou-se uma solução de hidróxido de sódio $(\mathrm{NaOH}) 0,5 \mathrm{M}$, sob agitação por $2 \mathrm{~h}$, a $60^{\circ} \mathrm{C}$, decorridas as duas horas a casca foi lavada com água destilada até alcançar a neutralidade e seca em estufa a $60^{\circ} \mathrm{C}$ por 24 horas (Mimura et al., 2010). O tratamento térmico foi realizado em mufla na ausência de oxigênio, onde o material foi submetido a uma temperatura de $600^{\circ} \mathrm{C}$ por três horas, com uma rampa de aquecimento de $5^{\circ} \mathrm{C} / \mathrm{min}$.

Ensaios em coluna de leito fixo: Os ensaios em coluna de leito fixo foram realizados variando a concentração inicial da solução de corante em 10,15 e $20 \mathrm{mg} . \mathrm{L}^{-1}$, vazão da alimentação em 4, 6 e $8 \mathrm{~mL}$. $\mathrm{min}^{-1}$, altura do leito de adsorção em 0,5, 1 e 1,5 cm em temperatura ambiente. Uma bomba peristáltica foi utilizada para alimentar a coluna a partir do reservatório da solução de corante. O fluxo da solução sintética de corante Verde Malaquita entra na base da coluna passando por todo leito e saindo no topo da coluna, onde é realizada a coleta da solução tratada já na sua concentração final. A coleta das amostras foi feita em intervalos 


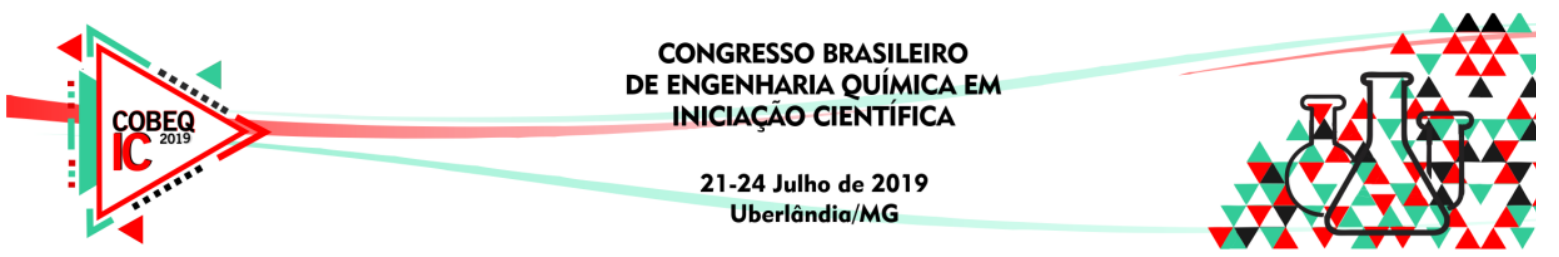

regulares de tempo. A partir do teor de corante presente nas amostras foram obtidas as curvas de ruptura $\left(\mathrm{C} / \mathrm{C}_{0} \mathrm{x}\right.$ t) (Carpiné, 2011).

Determinação dos parâmetros para avaliação fluidodinâmica: O desempenho dinâmico da adsorção em leito fixo depende de condições experimentais que afetam diretamente a resistência de transferência de massa e, portanto, os parâmetros de adsorção. Os seguintes parâmetros foram avaliados a partir das curvas de ruptura, a zona de transferência de massa (MTZ, cm) e a capacidade de adsorção do leito (Deokar e Mandavgane, 2015). É importante aplicar o balanço de massa na coluna para calcular as capacidades de adsorção no tempo de ruptura $\left(Q_{b}, m g \cdot g^{-1}\right)$ e saturação $\left(Q_{s}, m g \cdot g^{-1}\right)$ como fornecido na Equação 1:

$$
Q_{b, s}=\frac{C_{0} Q}{m} \int_{0}^{t_{b, s}}\left(1-\frac{C_{t}}{C_{0}}\right) d t
$$

Onde $t_{\mathrm{b}}(\min )$ e $\mathrm{t}_{\mathrm{s}}(\min )$ são os tempos de ruptura e saturação, respectivamente, $\mathrm{m}$ ( $\mathrm{g}$ ) é a massa do adsorvente, $\mathrm{Q}(\mathrm{L} . \mathrm{min}-1)$ é a vazão volumétrica da fase fluida, $\mathrm{C}_{\mathrm{o}}(\mathrm{mg} . \mathrm{L}-1)$ a concentração da solução sintética de corante na solução na alimentação da coluna e $C_{t}(m g$.L1) a concentração do adsorvato na solução na saída da coluna (Bordignon et al., 2013). Conhecidos os valores de $\mathrm{Q}_{\mathrm{b}}$ e $\mathrm{Q}_{\mathrm{s}}$ e com o valor da altura do leito (Z em centímetros) é possível calcular a zona de transferência de massa (MTZ) de acordo com a Equação 2:

$$
M T Z=Z\left(1-\frac{Q_{b}}{Q_{s}}\right)
$$

Na ZTM, a saturação do leito varia de 100 a $0 \%$ e a concentração de adsorvato varia da concentração de entrada a zero (solução afluente). O contato entre adsorvato e adsorvente expresso como tempo de contato do leito vazio (EBCT) ou tempo de residência, influencia o volume do afluente tratado e a natureza da curva de ruptura. A Equação 3 foi usada para determinar o tempo de residência (Srivastava et al., 2008).

$$
E B C T=\frac{A_{c} Z}{Q}
$$

Onde $\mathrm{A}_{\mathrm{c}}$ é a área da seção transversal da coluna.

\section{RESULTADOS E DISCUSSÃO}

\subsection{Parâmetros do leito fixo}

Na Tabela 1 estão listados os tempos de ruptura $\left(t_{b}\right)$ e saturação $\left(t_{s}\right)$, as capacidades de de adsorção no tempo de ruptura $\left(\mathrm{Q}_{\mathrm{b}}\right)$ e saturação $\left(\mathrm{Q}_{\mathrm{s}}\right)$, a zona de transferência de massa $(\mathrm{MTZ})$ e o tempo de residência (EBCT) determinados em cada um dos ensaios realizados na coluna de leito fixo. É possível observar que o EBCT é inversamente proporcional ao aumento da vazão e diretamente proporcional ao aumento da altura do leito. Quando a concentração do afluente é aumentada com vazão e altura do leito constantes, a saturação da coluna ocorre cedo devido a locais de adsorção limitados. Isto leva a uma redução nos valores de $t_{b}$ e $t_{s}$, gerando uma curva 


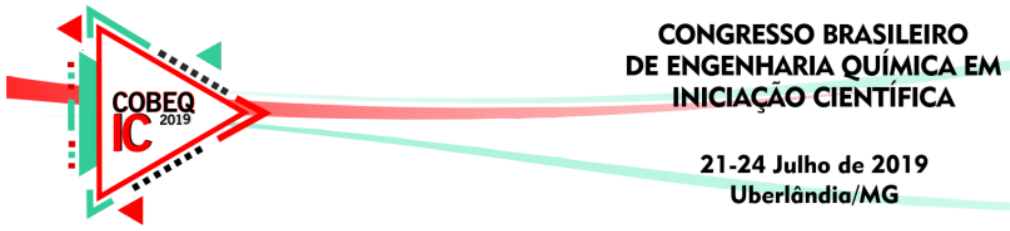

de ruptura mais acentuada em concentrações mais altas, como pode ser observado na Figura 2 (a). Nausheen et al., (2014) relataram um efeito semelhante para a remoção contínua do corante Vermelho Drimarine HF-3D na casca do amendoim.

Tabela 1 - Parâmetros calculados a partir das Equações (1), (2), (3) e (4)

\begin{tabular}{|c|c|c|c|c|c|c|c|c|c|}
\hline \multirow{2}{*}{ Parâmetros } & \multicolumn{3}{|c|}{$\begin{array}{c}\text { Variação de vazão } \\
\left(\mathrm{mL} \cdot \mathrm{min}^{-1}\right)\end{array}$} & \multicolumn{3}{c|}{ Variação da altura do leito } & \multicolumn{3}{c|}{ Variação de concentração } \\
& 4 & 6 & 8 & 0,5 & 1 & 1,5 & 10 & 15 & 20 \\
\hline & 20 & 14 & 20 & 18 & 20 & 20 & 154 & 36 & 20 \\
\hline $\mathrm{t}_{\mathrm{b}}(\mathrm{min})$ & 116 & 98 & 78 & 100 & 116 & 136 & 230 & 114 & 116 \\
\hline $\mathrm{t}_{\mathrm{s}}(\mathrm{min})$ & 7,95 & 16,29 & 30,94 & 13,89 & 7,95 & 5,33 & 30,29 & 10,39 & 7,95 \\
\hline $\mathrm{Q}_{\mathrm{b}}\left(\mathrm{mg} \cdot \mathrm{g}^{-1}\right)$ & 16,24 & 24,66 & 29,53 & 22,44 & 16,24 & 12,72 & 26,83 & 13,90 & 16,24 \\
\hline $\mathrm{Q}_{\mathrm{s}}\left(\mathrm{mg} \cdot \mathrm{g}^{-1}\right)$ & 0,51 & 0,34 & & 0,19 & 0,34 & 0,87 & & 0,25 & 0,51 \\
\hline MTZ $(\mathrm{cm})$ & 196,34 & 130,90 & 98,17 & 98,17 & 196,34 & 294,52 & 196,34 & 196,34 & 196,34 \\
\hline EBCT $(\mathrm{min})$ & $\left.196, \mathrm{~m}^{-1}\right)$ \\
\hline
\end{tabular}

\subsection{Curvas de Ruptura}

Efeito da variação da vazão: Na Figura 2 (a) pode-se observar as curvas formadas pela variação da vazão em 4, 6 e $8 \mathrm{~mL} \cdot \mathrm{min}^{-1}$ com concentração em $20 \mathrm{mg} \cdot \mathrm{L}^{-1}$ e a altura do leito em $1 \mathrm{~cm}$. Com o aumento da vazão há um declínio no tempo de saturação e no tempo de ruptura.

Efeito da variação da altura do leito: Na Figura 2 (b) as curvas formada pela variação da altura do leito na adsorção do verde malaquita pelo biochar. Variou-se a altura em $0,5,1$ e 1,5 $\mathrm{cm}$, mantendo constante a vazão de $4 \mathrm{~mL} \cdot \mathrm{min}^{-1}$ e a concentração inicial de $20 \mathrm{mg} . \mathrm{L}^{-1}$. Observase um ligeiro aumento no tempo de saturação com o aumento da altura do leito. A curva de avanço na Figura 2 (b) representa o aumento da transferência de massa devido ao aumento do EBCT com a altura do leito $(\mathrm{Z})$, consequentemente um maior volume de corante é removido. A Tabela 1 mostra o incremento em valores de $t_{\mathrm{s}}$ para valores de $\mathrm{Z}$ mais altos e aumento do MTZ. Com aumento do MTZ, há diminuição da resistência da transferência de massa. Em contraste, valores menores de MTZ para a menor altura do leito sugere uma utilização eficiente do adsorvente, o que facilitaria a regeneração fácil do adsorvente do ponto de vista energético. No entanto, o tempo de penetração é menor para a altura do leito menor devido à dispersão axial, que controla o mecanismo de transferência de massa (Safad et al., 2014).

Efeito da variação da concentração: A Figura 2 (c) mostra as curvas de ruptura para avaliação da variação da concentração inicial em 10,15 e $20 \mathrm{mg} \mathrm{L}^{-1}$, onde foram mantidas constantes a vazão e a altura do leito em $4 \mathrm{~mL} \cdot \mathrm{min}^{-1}$ e $1 \mathrm{~cm}$, respectivamente. Quando a concentração do afluente é aumentada e a vazão e altura do leito são mantidos constantes, a saturação da coluna ocorre cedo devido a locais de adsorção limitados. Isto leva a uma redução nos valores de $t_{b}$ e $t_{s}$, gerando uma curva de ruptura mais acentuada para concentrações mais 


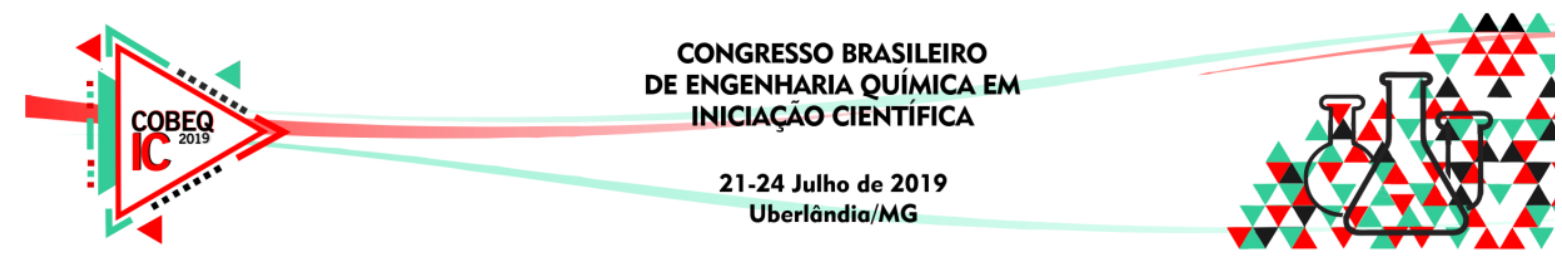

altas. O trabalho de Deokar e Mandavgane (2015) apresentou resultados semelhantes para remoção de Ácido 2,4-diclorofenoxiacético usando cinzas de casca de arroz.

Figura 2- Curvas de ruptura: (a) variando a vazão, (b) variando a altura do leito e (c) variando a concentração
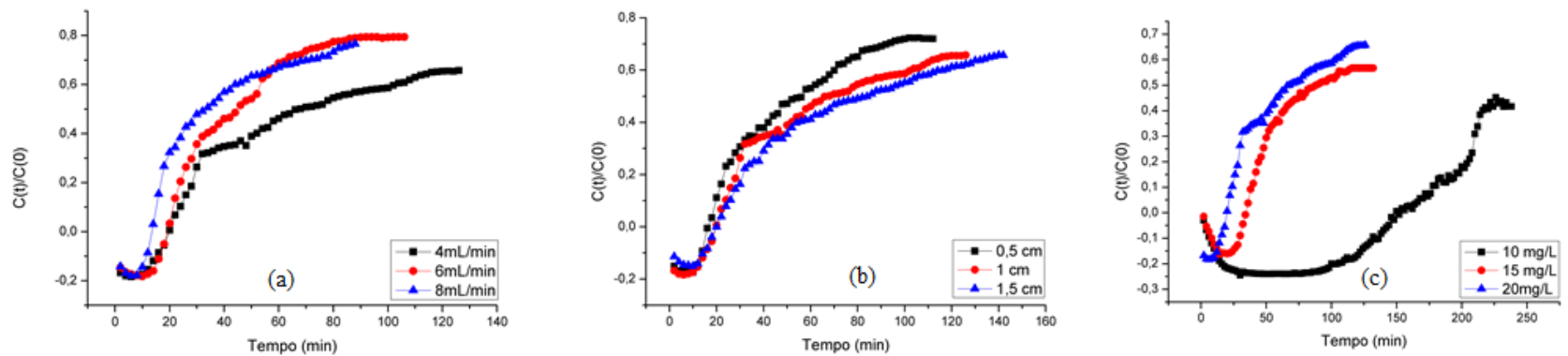

\section{CONCLUSÃO}

A eficiência de remoção do corante foi significativa, o que indica que o biocarvão proveniente da casca de arroz é um possível bioadsorvente alternativo no tratamento de efluentes que contenham esse corante. Através dos ensaios em coluna de leito fixo e das curvas de ruptura obtidas foi possível avaliar os efeitos causados pelas variações da concentração da solução de corante, da vazão de entrada e da altura do leito.

\section{REFERENCIAS}

ALEXANDER, D. et al. Investigation of simultaneous adsorption properties of $\mathrm{Cd}, \mathrm{Cu}, \mathrm{Pb}$ and $\mathrm{Zn}$ by pristine rice husks using ICP-AES and LA-ICP-MS analysis; Microchemical Journal, pag 3, 2017

BORDIGNON V.A.F., RIBEIRO C., BORBA C. E., SCHEUFELE F.B., Obtenção as curvas de ruptura de adsorção do corante reativo Azul 5G utilizando escamas do peixe Oreochromis niloticus, III Encontro Paranaense de Engenharia e Ciência, Paraná, 2013.

CARPINÉ, D. Recuperação do aroma álcool feniletílico por adsorção em batelada e em coluna. 115 p. Tese (Mestrado em Tecnologia de Alimentos) - Setor de Tecnologia de Alimentos, Universidade Federal do Paraná, 2011

DELLAMATRICE P.M., et al. Degradation of textile dyes by cyanobacteria. Braz. Jornal of Microb., 2016

DEOKAR S. K.; MANDAVGANE S. A., Estimation of packed-bed parameters and prediction of breakthrough curves for adsorptive removal of 2,4-dichlorophenoxyacetic acid using rice husk ash, Journal of Envir. Chem. Eng., 2015.

MANIQUE, M.C. Caracterização e utilização da cinza de casca de arroz como adsorvente na purificação de biodiesel de óleo de fritura. $54 \mathrm{f}$. Tese (Mestrado em Ciências dos 


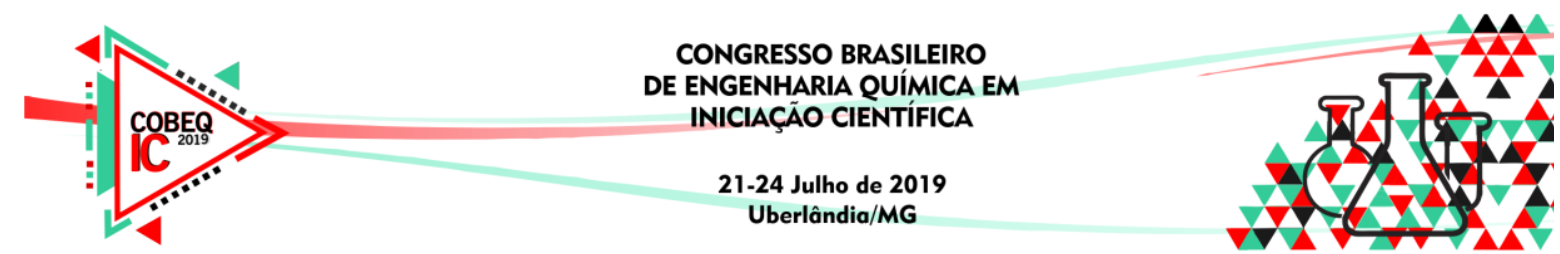

Materiais) - Programa de Pós Graduação em Ciências dos Materiais, Universidade Federal do Rio Grande do Sul, 2011.

MIMURA, A. M. S.; VIEIRA, T. V. de A.; MARTELli, P. B.; GORGULHO, H. de F. Aplicação da casca de arroz na adsorção dos íons $\mathrm{Cu}^{2+}, \mathrm{Al}^{3+}, \mathrm{Ni}^{2+}$ e $\mathrm{Zn}^{2+}$. Química Nova, v. 33, p. 1279-1284, 2010.

MONTANHER, S. F.; OLIVEIRA, E. A.; ROLLEMBERG, M. C. Removal of metal ions from aqueous solutions by sorption onto rice bran. Journal of Hazardous Materials, v. 117, p. 207-211, 2005.

NAUSHEEN, S.; BHATTI, H.N.; FURRUKH, Z.; SADAF, S.; NOREEN, S. Adsorptive removal of Drimarine Red HF_3D dye from aqueous solution using low-cost agricultural waste: batch and column study, Chem. Ecol. 30 (4) (2014) 376-392, 2014.

RIBAS, M. C. Remoção de corantes têxteis pelo processo de adsorção utilizando carvão ativado produzido a partir de subprodutos agroindustriais : estudos em batelada e coluna de leito fixo. 159 f. Tese (Doutorado em Engenharia Química), Universidade Federal do Rio Grande do Sul, 2016.

SAFAD S., BHATTI H.N., NAUSHEEN S., NOREEN S., Potential use of low-cost lignocellulosic waste for the removal of DiRecT violet 51 from aqueous solution: equilibrium and breakthrough studies, Arch. Environ. Contam. Toxicol. 66 (4) (2014) 557-571, 2014.

SANTHANAM, M., SELVARAJ, R., ANNAMALAI, S., SUNDARAM, M., Combined electrochemical, sunlight-induced oxidation and biological process for the treatment of chloride containing tetile effluent, Chemosphere, 2017

SRIVASTAVA, V. C.; PRASAD, B.; MISHRA, I. M.; MALL, I. D.; SWAMY, M. M. Prediction of 470 breakthr ough curves for sorptive removal of phenol by bagasse fly ash packed 471 bed. Ind. Eng. Chem. Res., v. 47 (5), p. 1603-1613, 2008.

SOUZA, N. K.; Adsorção de corantes catiônicos e aniônicos me solução aquosa usando matérias bi-funcionalizados a partir do bagaço de cana de açúcar; Universidade Federal de Ouro Preto, 2013.

THAKUR, V. K. Lignocellulosic Polymer Composites: Processing, Characterization and Proprieties. Wiley and Scrivener Publishing, New York, 2015. 